\title{
Effects of handling before central 6-hydroxydopamine treatment on subsequent emotionality and neurochemical changes in rats
}

\author{
DONALD V. COSCINA, JEFF GOODMAN, DAMODAR D. GODSE, and HARVEY C. STANCER \\ Section of Neurochemistry, Clarke Institute of Psychiatry \\ and
}

Departments of Psychology and Psychiatry, University of Toronto, Toronto, Ontario, Canada M5T 1R8

\begin{abstract}
Five minutes of daily handling 6 days prior to intracisternal 6 -hydroxydopamine (60HDA) treatment did not alter the magnitude or the time course of rage known to occur in rats after this method of chronic depletion of brain norepinephrine (NE) and dopamine (DA). However, handling produced an apparent protective effect against the ability of 6OHDA to deplete brain DA and, possibly, NE. Since previous work has shown that this same handling regimen, instituted after the production of $60 \mathrm{HDA}$-induced rage, has pronounced taming effects, these findings collectively show that handling is a potentially important variable to control in experiments concerned with the behavioral and neurochemical effects of this drug.
\end{abstract}

Intracisternal or intraventricular injection of 6-hydroxydopamine (6OHDA), sufficient to chronically deplete brain norepinephrine (NE) and dopamine (DA) in rats, can produce a syndrome of hyperemotional or hyperreactive behaviors operationally defined as "rage" (Coscina, Seggie, Godse, \& Stancer, 1973b; Nakamura \& Thoenen, 1972). We have recently reported (Coscina, Goodman, Godse, \& Stancer, 1975) that 5 min of daily handling, on Days 8-13 after central 6OHDA treatment, produces substantial diminution of this rage. The ameliorative effect of such handling was so prominent that we wondered if the same regimen prior to central 6OHDA administration would reduce or prevent such hyperemotional behaviors. To assess this possibility, we handled normal rats 5 min daily for 6 days before administering 6OHDA intracisternally and then followed the magnitude and time course of postinjection emotional responding.

\section{METHOD}

\section{Animals and Procedures}

Thirty-six male albino rats (Wistar strain, High Oaks Ranch, Ontario), weighing 320-350 g, were used. Rats were individually housed in a temperature $\left(72^{\circ} \mathrm{F} \pm 2^{\circ}\right)$ and light $(0800-2000 \mathrm{~h}$ on) controlled colony with food (on the floor of the cage) and water (in bottles with sipper tubes) available ad lib.

This research was supported by general funds from the Clarke Institute of Psychiatry. Portions of this work were presented at the 81st Annual Meeting of the American Psychological Association held in Montreal, Quebec in August 1973. The behavioral data reported were obtained by the second author as part of a B. A. honors thesis in psychology at the University of Toronto (1973). We gratefully acknowledge the excellent technical assistance of Rick Johnston and Peter Chan throughout various phases of this work. Send reprint requests to the first author at: Section of Neurochemistry, Clarke Institute of Psychiatry, 250 College Street, Toronto, Ontario, Canada M5T 1R8. Jeff Goodman's address is: Department of Ysychology, Graduate Division, York University, Downsview, Ontario, Canada.
Upon arrival in the laboratory, rats were randomly divided into "handled" and "unhandled" subgroups. For the next 6 days, 18 rats were handled, and 18 rats were left undisturbed in home cages. Handling consisted of placing each rat on a table top, allowing him brief (10-15 sec) exploration, picking him up around the body and holding him (3-5 sec), and repeating this sequence for the full $5 \mathrm{~min}$. Subsequent to handling treatments (1300-1500 h daily), 12 handled and 12 unhandled rats received one intracisternal injection of 250 micrograms (expressed as free base) $6 \mathrm{OHDA}-\mathrm{HCl}$ in 25 microliters of deionized water with $1 \%$ ascorbic acid added to retard oxidation (see Coscina et al., $1973 \mathrm{~b}$ for description of injection procedure). The remaining six handled and six unhandled rats were not injected since earlier work revealed neither behavioral nor neurochemical effects of vehicle treatment (Coscina et al., 1973b).

Following 60HDA treatment, all rats were weighed and rated on 0-4-point scales for resistance to capture, magnitude and frequency of startle responding on Days $4,10,17,24$, and 31 postinjection (see Coscina et al., 1973b for details of testing procedure and rating criteria). As in our previous work on the effects of handling on 6OHDA-induced "rage" (Coscina et al., 1975), all handling and ratings were performed by an experimenter blind to final group designations.

After behavioral testing was completed, rats were left undisturbed in home cages for 1 week and then killed rapidly by decapitation. Brains were removed and prepared as previously described (Coscina, Rosenblum-Blinick, Godse, \& Stancer, 1973a; Coscina et al., 1973b) prior to fluorometric determinations of endogenous forebrain NE, DA, serotonin (5-hydroxytryptamine or 5-HT) and 5-hydroxyindoleacetic acid (5-HIAA) levels. NE and DA were determined by the method of Shellenberger and Gordon (1971). 5-HT and 5-HIAA were determined by the method of Maickel, Cox, Saillant, and Miller (1968).

All behavioral and body weight data were analyzed by separated three-way analyses of variance with corrections for repeated measures and unequal sample sizes. Biochemical results were analyzed by $t$ tests for independent samples. All ps reported represent two-tailed distributions.

\section{RESULTS}

Of the 24 rats receiving 6OHDA, 4 died soon after injection and 1 was too ill to complete the entire experiment. Therefore, final group sizes were reduced to 

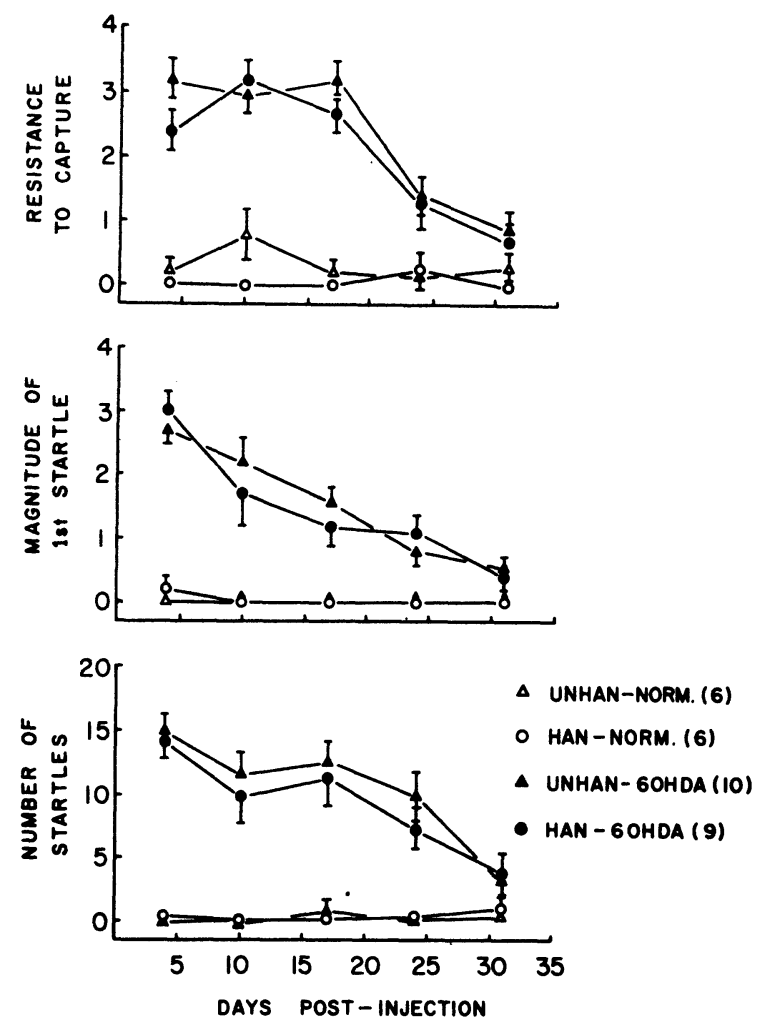

Figure 1. Means and standard errors of emotionality scores as described in the text depicting the time course of 60HDA-induced rage in handled or unhandled rats as compared to appropriate controls. Group designations and ns for each group are depicted in the figure.

10 and 9 for unhandled-60HDA and handled-60HDA animals, respectively.

Regardless of handling pretreatment, rats receiving 6OHDA showed elevated scores (ps $<.001)$ on all behaviors rated compared to controls (see Figure 1). The magnitude of these responses could not be distinguished as a function of prior handling. By way of contrast, normal rats showed minimal responding on all behaviors rated across the assessment period. Again, such responding could not be distinguished as a function of pretest handling. Regardless of handling, the body weights of 60HDA-treated rats were significantly lower $(p<.001)$ than those of normals (see Figure 2). However, all groups displayed significant weight gain (ps $<.01)$ over the experimental period.

Forebrain samples from handled as well as unhandled rats which received 60HDA contained significantly less (ps $<.001$ ) endogenous NE and DA when compared to their appropriate control groups (see Figure 3). In addition, small (12\%-14\%) but significant decrements (ps <.05) in forebrain 5-HT levels were also found. No differences in 5-HIAA or 5-HT/5-HIAA ratios were found between handled-60HDA and unhandled-60HDA rats. Of particular interest, DA depletion was less severe $(\mathrm{p}<.025)$ in handled vs. unhandled rats receiving

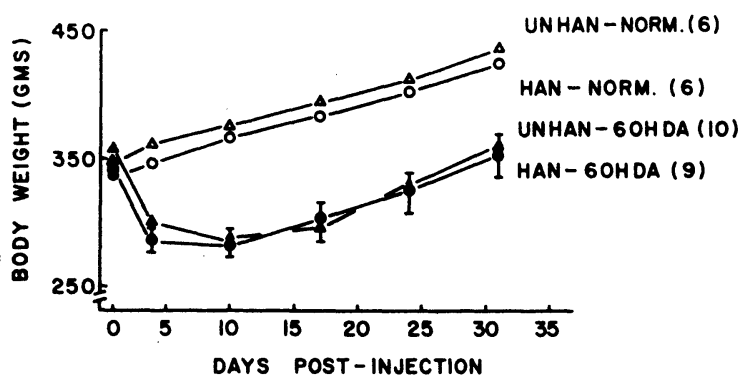

Figure 2. Means and standard errors of body weights for groups described in Figure 1.

60HDA. A similar but insignificant trend $(.10>p>.05)$ for NE depletion was observed between these groups as well. No differences were found between handled-normal and unhandled-normal groups on measures of forebrain NE, DA, 5-HT, or the ratio of 5-HT/5-HIAA. Levels of 5-HIAA were slightly higher $(p<.05)$ in samples from unhandled vs. handled normals.
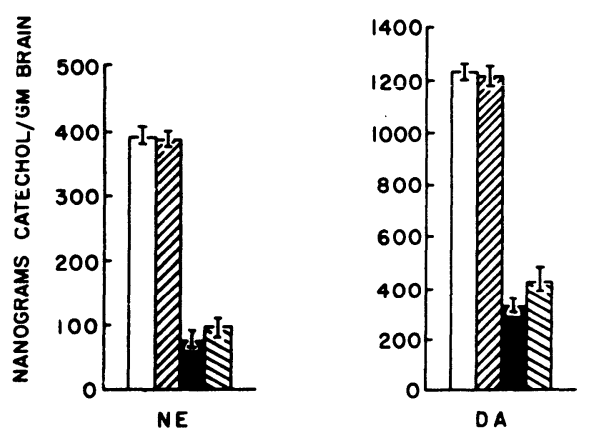

UNHANDLED, NORMAL (5)
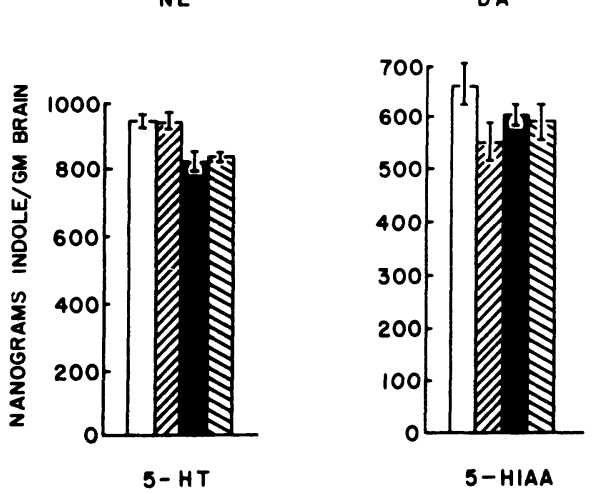

HANDLED, NORMAL (5) UNHANDLED, 6-OHDA (10)

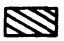

HANDLED, 6-OHDA (9)

Figure 3. Means and standard errors of forebrain levels of endogenous NE, DA, 5-HT, and 5-HIAA for groups described in Figure 1. Abbreviations for brain amines listed are defined in the text. Group designations and Ns for each group are depicted in the figure. Samples from one handled and one unhandled normal control were lost during assay procedures, hence the reduction in $\mathbf{N}$ compared to those indicated in Figures 1 and 2. 


\section{DISCUSSION}

Brief ( $5 \mathrm{~min}$ ) daily handling of normal rats for 6 days was ineffective in modifying either the magnitude or the time course of rage which followed central 6OHDA treatment. This finding was of interest since the same handling regimen instituted 1 week after central 6OHDA treatment is quite effective in curtailing hyperemotional responses (Coscina et al., 1975).

In previous work, we have pointed out the qualitative and quantitative similarities of both type and duration of rage induced by either central 6OHDA treatment or bilateral septal injury (Coscina et al., 1973a, b; 1975). Within the context of the present experiment, we know of no data which systemically show that handling prior to septal injury prevents or attenuates lesion-induced hyperemotionality. Preoperative testing of normal rats, which necessitates handling, for 6 days prior to septal lesioning does not prevent the appearance of septal rage (Yutzey, Meyer, \& Meyer, 1967). While there have been claims that extensive (e.g., 30 days) daily handling reduces the incidence of septal rage (see Fried, 1973, for review), our work shows (Coscina et al., 1975; present experiment) that the same amount of handling effective in curtailing 6OHDA-induced rage when instituted after central injection is ineffective when instituted prior to injection.

All surviving 6OHDA-treated rats, regardless of handling pretreatment, displayed marked hypophagia and hypodipsia proximal to drug administration which reflected in significant weight loss (see Figure 2). The debilitatory effects of central 60HDA treatment on consummatory behaviors are now well established (e.g., Stricker \& Zigmond, 1974) and have been observed by us before (Coscina et al., 1973a, b; 1975). The present observation of a seeming inverse relationship between indices of rage and body weight (Figures 1 and 2) might suggest to some that rage is an indirect consequence of generalized debilitation as reflected by weight loss. We feel such a conclusion to be erroneous. For one thing, weight loss induced either by food or water deprivation in normal rats or intake restrictions subsequent to lateral hypothalamic injury is not associated with rage (unpublished observations). Furthermore, re-examination of our behavioral data after factoring out variability associated with weight differences (analyses of covariance) still reveal significant 60HDA effects. That is, 6OHDA treatment per se was associated with production of rage as compared to control conditions. Therefore, we feel that the observed coincidence of 6OHDA-induced rage and weight loss represents a correlative, but not a causal, relationship.

Although preinjection handling did not alter the behavioral measures of 6OHDA-induced rage, such treatment produced apparent "protective" effects on forebrain DA system's susceptibility to 6OHDA's depletive action. A similar but insignificant trend was observed between these two groups for levels of forebrain NE. This effect did not reach statistical reliability, however, perhaps because measures of NE depletion in both groups approached the confidence limits of our assay, i.e., < two times blank values. It is not possible at this time for us to say whether this apparent effect of handling is a direct consequence of altered metabolic processes in central neural systems perse or if handling simply alters more basic physiological mechanisms (e.g., cardiac or respiratory functions) which themselves produce nonspecific changes that affect metabolism or clearance of 6OHDA from brain. Whatever mechanisms are responsible for this handling effect, our data show that handling is a potential variable which should be controlled in experiments employing this drug for biochemical-behavioral investigations.

The fact that a discrepancy could exit here between brain amine levels but not hyperemotional indices presents a situation opposite to that seen by us before (Coscina et al., 1975) in which postinjection handling produced behavioral, but not biochemical, changes. Taken together, these findings along with other work (Sorenson \& Ellison, 1973) suggest the lack of clear-cut relationships between whole-brain measures of monoamine metabolism and behavioral indices of emotionality. Perhaps future studies of this nature will evidence better behavioral correlates of emotionality with these neurochemical systems if more dynamic aspects of monoamine metabolism are explored (e.g., turnover rates, ratios of free/bound amines, changes in postsynaptic sensitivity or presynaptic uptake). Alternatively, more sensitive measures of emotionality changes in animals might produce more adequate scales to relate to biochemical measures. Regardless of the precise metabolic changes suspected to be responsible for the effects of handling on central 6OHDA-induced hyperemotionality (Coscina et al., 1975) or neurochemical changes (present experiment), these effects of such relatively mild and brief somato-sensory stimulation seem to merit further investigation.

\section{REFERENCES}

Coscina, D. V., Goodman, J., Godse, D. D., Stancer, H. C. Taming effects of handling on 6-hydroxydopamine induced "rage." Pharmacology, Biochemistry, and Behavior, 1975, 3, in press.

Coscina, D. V., Rosenblum-Blinick, C., Godse, D. D., \& STANCER, H. C. Consummatory behaviors of hypothalamic hyperphagic rats after central injection of 6-hydroxydopamine. Pharmacology, Biochemistry, and Behavior, 1973, 1, 629642. (a)

Coscina, D. V., Seggie, J., Godse, D. D., \& Stancer, H. C. Induction of rage in rats by central injection of 6-hydroxydopamine. Pharmacology, Biochemistry, and Behavior, 1973, 1, 1-6. (b)

Fried, P. A. The septum and hyper-reactivity: A review. British Journal of Psychology, 1973, 64, 267-275. 
Maickel, R. P., Cox, R. H., Saillant, J., \& Miller, F. P. A method for the determination of serotonin and norepinephrine in discrete areas of rat brain. International Journal of Neuropharmacology, 1968, 7, 275-281.

Nakamura, K., \& Thoenen, H. Increased irritability: A permanent behavior change induced in the rat by intraventricular administration of 6-hydroxydopamine. Psychopharmacologia, 1972, 24, 359-372.

Shellenberger, M. K., \& Gordon, J. H. A rapid, simplified procedure for simultaneous assay of norepinephrine, dopamine, and 5-hydroxytryptamine from discrete brain areas. Analytical Biochemistry, 1971, 39, 356-372.
Sorenson, C. A., \& Ellison, G. D. Nonlinear changes in activity and emotional reactivity scores following central noradrenergic lesions in rats. Psychopharmacologia, 1973, 32, 313-325.

STRICKER, E. M., \& Zigmond, M. J. Effects on homeostasis of intraventricular injections of 6-hydroxydopamine in rats. Journal of Comparative and Physiological Psychology, 1974, 86, 973-994.

Yutzey, D. A., Meyer, D. R., \& MeYer, P. M. Effects of simultaneous septal and neo- or limbic-cortical lesions upon emotionality in the rat. Brain Research, 1967, 5, 452-458.

(Received for publication March 7, 1975; revision accepted April 23, 1975.) 